\title{
Aportaciones de las Prácticas de Campo en la formación del profesorado de Biología: un problema de investigación y una revisión documental
}

\section{Contributions of Field Practices in Biology Teacher Training: A Research Problem and a Documentary Review}

\section{Elías Francisco Amórtegui Cedeño(1) Olga Mayoral García-Berlanga ${ }^{(2)}$ y Valentín Gavidia Catalán ${ }^{(2)}$}

(1) Programa de Licenciatura en Ciencias Naturales y Educación Ambiental. Universidad Surcolombiana (2) Departamento de Didáctica de las Ciencias Experimentales y Sociales. Universidad de Valencia

Resumen: Mostramos una aproximación al estado actual de conocimiento sobre las Prácticas de Campo en la formación de docentes de biología, a través de una revisión documental realizada sobre las publicaciones en revistas y congresos en educación en Ciencias Naturales y enseñanza de la Biología durante el periodo 2005-2015. La metodología que orientó esta investigación corresponde a un enfoque cualitativo-interpretativo, la estrategia de análisis documental y la modalidad de estado del arte. Analizamos un total de 29 publicaciones, destacando cuatro tendencias: Revisiones/reflexiones, Investigaciones sobre enseñanza-aprendizaje, Investigaciones de las Concepciones sobre prácticas de campo y Relación con el Conocimiento Profesional del Profesor. Mostramos sus principales características, problemas y poblaciones de estudio. Por último, indicamos la importancia de las Prácticas de Campo en la formación del profesorado de Biología, específicamente las concepciones que se tienen sobre su diseño, desarrollo y evaluación.

Palabras clave: Práctica de Campo, Enseñanza de la Biología, Formación Docente, Conocimiento Profesional del Profesor.

Abstract: We show an approximation to the current state of knowledge about Field Practices in relation to biology teachers training, through a documentary review on publications in journals and conferences of Natural Sciences and Biology teaching during the period 2005-2015. The methodology that guided this research corresponds to a qualitative-interpretative approach, the strategy of documentary analysis and the modality of state of the art. We analyzed a total of 29 publications, of which we defined four tendencies: Revisions/reflections, Research on teaching-learning, Research on Conceptions on field practices and Relationship with Teacher's Professional Knowledge. We show their main characteristics, problems and study populations. Finally, we indicate the importance of Field Practices in the training of Biology teachers, specifically the conceptions that have their design, development and evaluation.

Keywords: Field Practice, biology education, teacher training, teacher professional knowledge.

(Fecha de recepción: marzo, 2017, y de aceptación: junio, 2017)

DOI: 10.7203/DCES.32.9940 


\section{Introducción}

Durante mucho tiempo, la profesión docente se ha subestimado al considerar que para enseñar basta con tener dominio del conocimiento disciplinar en profundidad, dejando de lado el hecho de que los docentes poseen un conocimiento particular que incluye aspectos didácticos, pedagógicos, del contexto, entre otros, que les posibilita enseñar adecuadamente (Gil et al, 1991; Valbuena, 2007). Esta situación parece específica de la profesión docente y diferente a lo que ocurre con los profesionales de otras áreas (Bromme, 1988), en los que todos los aspectos relativos a la profesión son muy cuidados y puestos en valor, como es el caso de médicos, abogados, etc. Este escenario viene acompañado, en muchos países, de una baja valoración social y escasa formación requerida para ejercer la docencia (Tardif y Lessar, 2014). Por otra parte, diversas investigaciones reconocen el Conocimiento Didáctico del Contenido (CDC) como núcleo del Conocimiento profesional del docente (Shulman 1986, 1987; Carlsen, 1999; Park y Chen, 2012; Gess-Newsome, 2015), integrado por diversos componentes, uno de los cuales hace referencia a las estrategias de enseñanza, en el que ubicamos las Prácticas de Campo.

En términos generales, las Prácticas de Campo pueden considerarse como una estrategia de enseñanza en las ciencias naturales donde los alumnos han de utilizar procedimientos, habilidades y destrezas específicas para resolver problemas de carácter científico relativos a la Biología y/o Geología (Del Carmen, 2000, Del Carmen, 2011) que favorecerán aspectos como los siguientes (Caamaño, 2003):

- La integración de la teoría con la práctica.

- La comprensión sobre la construcción del conocimiento científico.

- La adquisición de habilidades científicas: observar, clasificar, interpretar fenómenos, plantear y contrastar hipótesis y extraer conclusiones (procesos cognitivos); el manejo adecuado de instrumentos de medición, manejo de variables, realización de cálculos (estrategias de investigación).

- La potenciación de la motivación hacia las ciencias experimentales y la generación de actitudes positivas hacia la ciencia, tales como la curiosidad, el trabajo en equipo, la indagación y la divulgación del conocimiento.

Consideramos que las Prácticas de Campo adquieren un valor especial en la enseñanza y aprendizaje de la Biología pues permiten al alumnado abordar su objeto de estudio, "lo vivo", lo más cerca posible a sus condiciones naturales, con una perspectiva sistémica y holística que les permite comprender la relaciones que conforman el fenómeno viviente en conjunto con su ambiente: redes tróficas, adaptaciones, relaciones inter e intra específicas, biodiversidad, 
ecosistemas, autopoiesis, etc. Pero además ofrecen oportunidades educativas de alto valor relacionadas con aspectos procedimentales y actitudinales, como son la apreciación del significado de naturaleza, la valoración, conservación, disfrute sostenible de los recursos naturales, etc.

Una de las preguntas clave que nos hacemos al organizar nuestro Conocimiento Biológico es ¿Qué relaciones tienen los seres vivos entre sí y con su medio? Es evidente que los tópicos que debemos tratar para su respuesta son más fácilmente abordables a través de las Salidas de Campo, al conferir una perspectiva sistémica a lo vivo in situ difícilmente alcanzable sin las relaciones directas entre individuos y entornos.

Gagliardi (1986) afirma que la Biología y su enseñanza pueden organizarse a partir de conceptos estructurantes (aquellos que permiten al estudiante transformar su sistema cognitivo y así, construir otros saberes, organizar los datos de diversas formas y modificar sus conocimientos previos). Reconoce como conceptos estructurantes la dominancia del nivel macroscópico sobre el microscópico, los sistemas jerárquicos de restricciones múltiples y la autopoiesis. Castro y Valbuena (2007) proponen además:

- La organización, entendida como un sistema de relaciones articuladas en los seres vivos (Jacob, 1999)

- La reproducción, como la forma de extender y mantener un determinado código genético.
- El sistema, dirigido a comprender el fenómeno viviente como biosistemas abiertos (Mahnner y Bunge, 2000).

- La diversidad biológica, como evidencia de las potencialidades de expresión de un determinado genotipo en diferentes ambientes.

En todos estos conceptos estructurantes, apreciamos una aportación fundamental de las Prácticas de Campo, pues permitirá al estudiantado comprender la variedad de formas y procesos que se llevan a cabo en el mundo viviente desde una perspectiva sistémica y holística centrada en una red de relaciones, abordando así los diferentes ecosistemas existentes en la biósfera.

Debemos tener en cuenta las dificultades de aprendizaje de la Ecología que vienen reflejadas (Banet, 2000; Jiménez, 2003) en la noción de ecosistema como el cúmulo de seres vivos, la percepción lineal de las relaciones, la concepción estática de su funcionamiento, la inadecuada interpretación de redes alimentarias y la escasa sensibilización sobre el impacto del ser humano. A esto debemos sumar que Bermúdez et al (2014) manifiestan que los libros de texto tratan la temática de la diversidad biológica de manera descontextualizada del ámbito social, aun cuando la dimensión ética y política de la biodiversidad es consustancial a su carácter científico y social.

Con base en todo lo anterior, consideramos que las Prácticas de Campo son fundamentales para superar estas 
deficiencias, ya que pueden movilizar el aprendizaje del alumnado y facilitar al docente una transposición didáctica más efectiva. Asimismo, le encontramos un valor añadido ya que el docente proporciona al estudiantado, además de una experiencia directa con los fenómenos naturales y la vida silvestre, la posibilidad de generar comportamientos pro ambientales y conciencia sobre aspectos de la conservación.

Sin embargo y a pesar de su importancia, destacamos que los estudios sobre la problemática de la utilización de los trabajos de campo en la formación del profesorado son escasos. El estudio de Correa y Valbuena (2012) sobre los problemas abordados en las investigaciones sobre Trabajos Prácticos durante el periodo 2004-2006 y el estudio de Valbuena, Correa y Amórtegui (2012) sobre la sistematización de artículos publicados sobre enseñanza de la Biología publicados durante el periodo 2007-2008, han puesto de manifiesto la predominancia de la investigación didáctica sobre el trabajo de laboratorio y la escasez de estudios sobre el trabajo de campo.

\section{Metodología}

La metodología que orienta esta investigación corresponde a un enfoque cualitativo-interpretativo, empleando la estrategia de análisis documental y la modalidad de estado del arte, destinada a proporcionar una síntesis crítica del conocimiento acumulado y acerca del nivel de comprensión que se tiene del objeto de estudio (Jiménez, 2006).
Se busca establecer un objeto de estudio, la clasificación de la información sobre el mismo y la construcción de categorías a partir del saber acumulado (Calvo, 1995).

Desarrollamos la investigación en tres fases: contextualización, clasificación y categorización. En la contextualización delimitamos el problema, identificamos las fuentes de información y seleccionamos las publicaciones para su sistematización. En la fase de clasificación elaboramos los resúmenes analíticos educativos (RAE); éstos son instrumentos en los que se busca expresar por escrito y de manera simplificada la información contenida en un texto. En la fase de categorización, sistematizamos la información con base en la tipología de RAE propuesta por Valbuena, Correa y Amórtegui (2012), quienes proponen como elementos de análisis de las publicaciones, la composición de equipo de trabajo, la temática biológica, la sección de la revista, los autores, las fuentes bibliográficas, la población objeto/sujeto de estudio, la metodología de estudio, el problema de investigación, entre otros.

En nuestro caso analizamos 29 artículos relacionados con las prácticas de campo en la enseñanza de la Biología y mostramos sus principales problemas de investigación, poblaciones sujeto/objeto de estudio y metodología de estudio.

Nuestra muestra está formada por las revistas sobre educación en ciencias naturales de diversos países aparecidas entre los años 2005 y 2015 , dando un total de 29 (Cuadro I), y los eventos 
tanto europeos como latinoamericanos sobre educación en ciencias naturales y enseñanza de la Biología (Cuadro II). Incluimos en la muestra los trabajos de Cutter (1993), Mick (1996), Manzanal, Rodríguez y Casal (1999) y Rodrigo et al (1999), dada su relevancia destacada en el reporte realizado en Amórtegui y Correa (2012) como estudios específicos sobre el trabajo de campo en la enseñanza de la Biología y/o la formación del profesorado de esta disciplina. Del universo de revistas, tres corresponden específicamente a la enseñanza de la Biología (Journal of Biological Education, The American Biology Teacher y Revista Biografía: Escritos sobre la Biología y su enseñanza).

Para la selección inicial del material hemos revisado en particular los apartados de Abstract y Keywords. Debemos aclarar que los trabajos denominados out-door of the school, tales como la visita a museos, zoológicos, jardines botánicos o centros interactivos de ciencias naturales, no los hemos tomado como Prácticas de Campo, pues consideramos que son actividades que, aunque conforman algunas de las posibilidades de la educación primordialmente no formal del alumnado, sus finalidades, orientaciones, enfoques, papel del docente e intervención con el objeto de estudio (en este caso, el fenómeno de lo vivo), se presenta de forma artificial.

\section{Resultados}

$\mathrm{Al}$ realizar el análisis de las 29 publicaciones seleccionadas en la muestra, hemos identificado cuatro grandes tendencias con relación al Problema de investigación que se aborda en las investigaciones sobre Prácticas de Campo en la enseñanza de la Biología: Revisiones-reflexiones (4 artículos; 13,8\%), Investigaciones sobre Prácticas de Campo en la enseñanza-aprendizaje (11; 38\%), Investigaciones sobre concepciones acerca de las Prácticas de Campo (9; 31\%) e Investigaciones sobre las Prácticas de Campo y su relación con el Conocimiento del Profesor (5; 17,2\%). A continuación mostramos las características de estas tendencias en cuanto a sus principales metodologías de estudio, población sujeto / objeto de estudio y algunos de sus hallazgos, incluyendo algunos ejemplos.

\section{Revisiones/reflexiones}

En esta tendencia se encuentran principalmente trabajos en los que se realizan revisiones sobre el trabajo de campo o reflexiones sobre su importancia en la enseñanza de la Biología. Aquí se encuentran los trabajos de Cutter (1993), Mick (1996), Lock (2010) y Rodríguez y Amórtegui (2012).

Los tres primeros corresponden al contexto anglosajón, específicamente a Reino Unido. Cutter (1993) y Lock (2010) realizan revisiones sobre la inclusión de los trabajos de campo en los currículos de educación primaria, secundaria y universitaria, tanto de Biología como de Geología. En el caso de Mick (1996) y Rodríguez y Amórtegui (2012), las reflexiones versan sobre 
el trabajo práctico de campo en Biología, y su importancia en la enseñanza del concepto de Biodiversidad. Sólo en el trabajo de Lock (2010) encontramos una postura metodológica concreta, compuesta por la revisión documental, el estudio histórico y el análisis de contenido, además de un problema concreto de investigación y un tratamiento de resultados.

En esta tendencia se encuentran investigaciones concisas y claras metodológicamente (enfoques mixtos, empleo de cuestionarios, observación participante, entre otros), que presentan problemas y objetivos de investigación, así como el diseño de instrumentos de recolección de información y el tratamiento de resultados.

\section{Investigaciones sobre Prácticas de}

Campo en la enseñanza-aprendizaje

Esta tendencia se centra en investigar los resultados del trabajo de campo en el aprendizaje de la Biología, sistematizando su impacto en la enseñanza-aprendizaje de la Biología, tanto en educación primaria, como secundaria y universitaria. Aquí tenemos los trabajos de Manzanal, Rodríguez y Casal (1999), Anderson, Thomas y Nashon (2008), Legarralde, Vilches y Darrigran (2009), Grandi y Motokane (2009), Judson (2011), Morag y Tal (2012), Gómez (2014), Tal, Lavie Alon y Morag (2014), Lavie Alon y Tal (2015), Flórez y Gaitán (2015) y Guarnizo, Puentes y Amórtegui (2015).
Manzanal, Rodríguez y Casal (1999) y Legarralde, Vilches y Darrigran (2009) se han centrado en la contribución del trabajo de campo en estudiantes de secundaria españoles y de carreras biológicas de Argentina respectivamente, concluyendo que éste permite la comprensión de los conceptos y principios de la Ecología y la generación de actitudes más favorables en la defensa de los ecosistemas, así como la adquisición de procedimientos y habilidades científicas, desde las más básicas (utilización de aparatos, medición, tratamiento de datos) hasta otras más complejas (investigar y resolver problemas haciendo uso de la información obtenida).

En el contexto israelí los estudios se han realizado tomando como referencia el Field Trip in Natural Enviroments (FiNE), propuesta basada en el marco teórico sobre el aprendizaje en el campo y la educación ambiental planteado por Storksdieck (2006). En tal sentido Morag y Tal (2012) analizan 22 salidas a parques naturales con estudiantes de $4^{\circ}$ y $6^{\circ}$ grado de diversas escuelas y condiciones socio-económicas entre 2006 y 2009. El análisis de las observaciones de campo y las entrevistas pre y post a 41 estudiantes mostraron que durante las actividades predomina el uso de pedagogías tradicionales y se resalta la importancia de las interacciones sociales y físicas en el aprendizaje. Tal, Lavie Alon y Morag (2014) realizan el análisis de 62 salidas de campo con estudiantes de entre 10 y 14 años, y concluyen que existen cuatro elementos que constituyen una práctica de campo de alta 
calidad: actividad-acción, involucramiento de profesores, uso del entorno y el aprendizaje social. Lavie Alon y Tal (2015) estudian varias características de las salidas, tales como su preparación, su desarrollo y su conexión con el currículo. Los estudiantes alcanzan su aprendizaje en tres aspectos: el cognitivo, el afectivo y el comportamental.

En el contexto de Norteamérica, Anderson, Thomas y Nashon (2008), realizan un seguimiento de una salida realizada por estudiantes de Biología en secundaria (15-16 años) de una escuela de Canadá a una reserva ecológica protegida, en la cual debían trabajar en grupos y realizar recorridos por diversas áreas biológicas. El estudio se centró en analizar el rol metacognitivo de los estudiantes dentro de cada grupo de trabajo, concluyendo que existen factores metasociales y metacognitivos que influyen en la efectividad del aprendizaje de las ciencias. Por su parte, Judson (2011) estudia la forma en la que el trabajo de campo puede promover la construcción de modelos mentales sobre el ambiente desértico en estudiantes de $4^{\circ}$ y $7^{\circ}$ grado (9-13 años) al visitar The Sonoran Desert Center en Estados Unidos.

En un análisis de 25 estudiantes de séptimo grado de una escuela en Brasil, Grandi y Motokane (2009) destacan la importancia de la vinculación del alumnado en el diseño de las guías de campo, permitiendo generar mayores elementos argumentativos en la comprensión de las actividades desarrolladas en campo.
En el contexto colombiano resaltamos las propuestas realizadas por Gómez (2014) y Guarnizo, Puentes y Amórtegui (2015). El trabajo de Gómez (2014) se centra en la Investigación como Estrategia Pedagógica y aborda la promoción de actitudes, conocimientos y prácticas hacia la conservación del ecosistema de páramo en estudiantes de educación secundaria. El estudio de Guarnizo, Puentes y Amórtegui (2015) muestra el impacto de las salidas de campo en el aprendizaje de contenidos conceptuales sobre la riqueza y abundancia biológica, la morfología vegetal, la taxonomía y clasificación de plantas, así como la adquisición de habilidades y destrezas relacionadas con el tratamiento de material biológico y la generación de actitudes hacia la identidad del alumnado con su región.

Investigaciones sobre concepciones acerca de las Prácticas de Campo

En esta tendencia encontramos investigaciones cuyo objeto de estudio son particularmente las concepciones (Percepciones u Opiniones) y mostramos tres agrupaciones teniendo en cuenta la población de estudio: Alumnado, Profesorado en Ejercicio y Profesorado en Formación.

Para el caso del alumnado encontramos el trabajo de Goulder, Scott y Scott (2013) centrado en 54 estudiantes de un curso previo a la carrera de Biología en la Universidad de Hull (Reino Unido). Concluyeron que los factores demográficos/personales tienen poca influencia 
Cuadro I. Revistas sobre educación en Ciencias Naturales revisadas (en negrita aquellas exclusivas sobre enseñanza de la Biología)

\section{$\mathrm{N}^{\circ}$ REVISTA}

$1 \quad$ The American Biology Teacher

2 Journal of biological education

3 Revista Biografía: Escritos sobre la Biología y su enseñanza

4 Journal of science teacher education

5 Science teacher

6 Journal of research in science teaching

$7 \quad$ Electronic journal of science education

8 Journal of College Science Teaching

9 Science Education

10 International journal of science education

11 Studies in Science Education

12 Science \& education

13 Teaching Science

14 Research in science education

15 The Journal of Environmental Education

16 Connect UNESCO international science, technology and environmental education newsletter

17 Educación y Ciencia

18 Journal of science education and technology

19 Journal of Baltic Science Education

20 International Journal of Science and Mathematics Education

21 Journal of Turkish Science Education

22 Enseñanza de las ciencias

23 Revista Electrónica de Enseñanza de las ciencias

24 Revista Didáctica de las Ciencias Experimentales y Sociales

25 Alambique: Didáctica de las Ciencias Experimentales

26 Revista Eureka sobre Enseñanza y Divulgación de las Ciencias

27 Investigación en la escuela

28 Revista TED: Tecné, Episteme y Didaxis

29 Ciência y educação 


\section{Cuadro II. Eventos nacionales e internaciones revisados sobre enseñanza de las ciencias naturales}

\section{Título del evento académico}

Año

VIII Encuentro Nacional de Experiencias en Enseñanza de la Biología y la Educación Ambiental y III Congreso Nacional de Investigación en Enseñanza de la Biología.

$\mathrm{X}$ Encontro Nacional de Pesquisa em educação em ciências.

VIII Congreso Iberoamericano de Educación Científica - II Congreso Internacional de Pedagogía, Didáctica y TIC aplicadas a la educación.

11th Conference of the European Science Education Research Association (ESERA).

VI Congreso Internacional de Formación de Profesores de Ciencias

Cuarto Congreso Nacional de Investigación en Educación en Ciencias y Tecnología.

2015. Bogotá (Colombia)

2015. Lindoia (Brasil)

2015. Bogotá (Colombia)

2015. Helsinki

(Finlandia)

VII Encuentro nacional de experiencias en enseñanza de la Biología y la Educación Ambiental y II Congreso nacional de investigación en enseñanza de la Biología.

IX Congreso internacional de Didáctica de las ciencias.

Tercer Congreso Nacional de Investigación en Educación en Ciencias y Tecnología.

2014. Bogotá (Colombia)

2014. Manizales (Colombia)

X Jornadas Nacionales y V Congreso Internacional de Enseñanza de la Biología.

2013. Bogotá

(Colombia)

XXV Encuentro de Didáctica de las Ciencias Experimentales.

2013. Girona (España).

2012. San Juan de Pasto (Colombia)

2012. Córdoba

(Argentina)

2012. Santiago de Compostela (España).

VI Encuentro nacional de experiencias en enseñanza de la Biología y la Educación Ambiental y I Congreso nacional de investigación en enseñanza de la Biología.

V Congreso Internacional de formación de profesores de ciencias.

2011. Bogotá (Colombia)

XXIIIV Encuentros de Didáctica de las Ciencias Experimentales-APICE.

II Congreso Internacional de DIDÁCTICAS-Universidad de Girona.

IX Jornadas Nacionales y IV Congreso Internacional de Enseñanza de la Biología.

Segundo Congreso Nacional de Investigación en Educación en Ciencias y Tecnología.

2011. Bogotá (Colombia)

2010. Jaén (España)

2010. Girona (España)

2010. San Juan de Tucumán (Argentina)

IV Congreso Internacional de Formación de Profesores de Ciencias.

2010. Santiago de Cali (Colombia)

VIII Congreso Internacional sobre Investigación en la Didáctica de las Ciencias.

2009. Bogotá

(Colombia)

International Congress of Science Education, 10 Years of The Journal of Science Education.

2009. Barcelona (España)

2009. Cartagena

(Colombia) 
sobre su apreciación acerca del trabajo de campo; sin embargo el alumnado que tuvo una experiencia negativa en el trabajo de campo en su educación secundaria, tenía menor aprecio a las salidas de campo en la educación superior.

Sobre el profesorado hemos encontrado gran diversidad de estudios a nivel internacional (España, Portugal, Brasil, Venezuela), siendo las concepciones del profesorado de ciencias naturales (generalmente de Biología y Geología) el objeto de estudio más abordado. Los trabajos de Tilling (2004), Dourado (2006), Nunes y Dourado (2009), Berezuki, Obara y Silva (2009), Stolpe y Björklund (2012) y Del Toro (2014) se han centrado en las concepciones del profesorado en activo. Las metodologías de estos estudios han sido tanto cuantitativas como cualitativas, empleado mayoritariamente cuestionarios aplicados en una sola ocasión.

En general los docentes atribuyen al trabajo de campo grandes cualidades, pero no las aplican en sus prácticas docentes, ya sea por la cultura escolar o por el desconocimiento didáctico para abordarlas (Lock, 2010; Graham et al, 2015).

Llama la atención el trabajo de Stolpe y Björklund (2012) quienes analizan la capacidad de dos docentes de Ecología suecos de atender a detalles esenciales en un complejo ambiental durante una salida de campo. Aquí usan grabaciones de video y entrevistas en campo para sistematizar la salida de campo realizada.

Sobre los estudios centrados en la formación inicial docente (la menos fre- cuente) en forma de Máster, o de estudios de grado encontramos los trabajos de Rodrigo et al (1999) y Ríos y Rueda (2009), que consideran fundamentales los trabajos de campo en la formación del futuro docente. Sin embargo sus concepciones son más diversas que en las de profesorado en ejercicio, pues algunos consideran importante desarrollar salidas de tipo investigativo y vincularlas con el trabajo previo y posterior de la clase, y otros mantienen ideas del trabajo de campo donde prima el papel activo del docente y el pasivo del alumnado.

Investigaciones sobre la relación de las Prácticas de Campo con el Conocimiento Profesional del Profesor

En esta última tendencia hemos considerado aquellas investigaciones que han abordado los Trabajos de Campo en la formación de docentes de Biología, explicitando las implicaciones de sus concepciones y prácticas docentes en la construcción de su Conocimiento Profesional, su Conocimiento Didáctico y desarrollo docente. La línea de investigación sobre el Conocimiento Profesional de Profesor se ha convertido en un referente para comprender el quehacer docente (Abell, 2008).

Aunque algunos de estos trabajos podrían ubicarse en el apartado anterior sobre las Concepciones de las Prácticas de Campo en la formación inicial de los futuros docentes, consideramos que en el abordaje que se hace en esta línea de estudios, se resalta principalmente la importancia de las Prácticas 
de Campo el profesor como sujeto de conocimiento docente.

Aquí encontramos los trabajos de Tal y Morag (2009), Amórtegui y Correa (2012), Sánchez y Escobar (2014), Amórtegui (2014) y Costillo et al (2014). En esta perspectiva los enfoques metodológicos han sido principalmente cualitativos, a través del empleo del análisis de contenido. Amórtegui y Correa (2012) han estudiado el impacto de las Prácticas de Campo en un programa de formación inicial de decentes de Biología, en la construcción del conocimiento del profesor, específicamente desde su aporte en el conocimiento del contenido y en el conocimiento didáctico del contenido.

Por otra parte, Amórtegui (2014) se ha centrado en las concepciones sobre las salidas de campo en futuros docentes de Ciencias Naturales de la Universidad Surcolombiana, evidenciando que para estos docentes en formación, en las actividades de campo prima la reafirmación de la teoría con la práctica, y la enseñanza-aprendizaje de contenidos conceptuales y procedimentales particularmente. Aquí se realiza un análisis de sus implicaciones en la construcción del Conocimiento Profesional del Profesor.

El trabajo de Tal y Morag (2009) analiza la importancia de la reflexión, elemento fundamental en el Conocimiento Didáctico del Contenido, como un aspecto relevante en la formación y ejercicio docente en el momento de trabajar las salidas de campo; a través del diseño de materiales, sesiones de enseñanza y actividades de reflexión sobre el uso del Ecological Garden (EG) en
Israel. El análisis de tres docentes en formación inicial sin experiencia y dos profesores en ejercicio nóveles, muestra que las Prácticas de Campo favorecidas por la reflexión de la práctica individual y en grupo puede potenciar que los docentes lleven a cabo actividades de campo para alumnos de escuelas secundarias que visitaban el EG.

Por último, Costillo et al (2014) analizan las Prácticas de Campo de 48 profesores en formación de Educación Secundaria cursando el Máster Universitario en Formación del Profesorado en Educación Secundaria de la Universidad de Extremadura en la Especialidad de Biología y Geología. Los resultados mostraron que éstas eran excesivamente teóricas, centradas en el profesor y en determinados casos sin relación con las clases. El estudio permitió a los docentes en formación hacer una reflexión personal sobre el diseño de sus salidas de campo, entendiendo que para mejorarlas se debía dar un papel más activo al alumnado, en el marco del Conocimiento Profesional y el desarrollo docente.

\section{Conclusiones}

Consideramos que las Prácticas de Campo son fundamentales en la enseñanza y aprendizaje de conceptos, procedimientos y actitudes en la Biología, pues permiten al alumnado abordar de manera especial el fenómeno viviente en los entornos naturales, específicamente desde una perspectiva sistémica y holística que lleve al estudiantado a 
considerar los ecosistemas como redes de relaciones y a generar actitudes en la conservación de la diversidad biológica.

Por ello, el profesorado de Biología debe tener una formación adecuada y suficiente para saber qué enseñar sobre la naturaleza y cómo enseñar en ella, de tal forma que se pregunte por qué debe aprender el alumnado en el campo, qué relaciones establecer entre la teoría y la práctica, cómo vincular las Prácticas de Campo al currículo de Ciencias, cómo evaluar el aprendizaje del alumnado, desde qué enfoque didáctico orientar la actividad y cuál es el aporte de la salida de campo a su desarrollo profesoral, sabiendo diseñar, poner en marcha, establecer relaciones con otras materias y evaluar este tipo de actividades. Esto pone en evidencia la necesidad de un programa de formación docente que favorezca el desarrollo profesoral sobre el adecuado conocimiento de contenido y didáctico requerido para enseñar Biología a través de las Prácticas de Campo.

Sin embargo, la revisión que hemos realizado pone en evidencia la escasez de estudios sobre la contribución del diseño de Prácticas de Campo en la construcción del Conocimiento Profesional del Profesor en la formación inicial docente. Pero además destacamos la necesidad de investigar con mayor profundidad las concepciones del profesorado en su formación inicial, pues es donde existe mayor desconocimiento sobre la importancia del trabajo de campo en la enseñanza de la Biología.

Por último, consideramos que para sacar el máximo provecho de esta estra- tegia, debe existir una formación inicial y continuada sobre el aprovechamiento de este tipo de actividades, que aborde el qué, el cómo y el por qué enseñar y aprender en el campo. La literatura aquí reportada muestra que se ha estudiado principalmente la relación teoría-práctica, las finalidades de aprendizaje, el rol del docente y el alumno, el momento de realización y la evaluación del aprendizaje, todas en el marco del desarrollo de Prácticas de Campo; sin embargo, añadimos que es fundamental además investigar, reflexionar y concretar específicamente sobre la naturaleza del Trabajo de Campo, qué lo diferencia de otras estrategias de enseñanza-aprendizaje, cuál es su relación con la epistemología de la Biología como disciplina científica, cómo planificarlo (antes, durante y después de su realización), qué tener en cuenta en una guía de campo y finalmente, cuál es su contribución a la construcción del conocimiento del profesor.

\section{Referencias bibliográficas}

ABELL, S. (2008). Twenty Years Later: Does Pedagogical Content Knowledge remain a useful idea? International Journal of Science Education. 30 (10), 1405-1416. DOI: $10.1080 / 09500690802187041$.

AMÓRTEGUI, E. (2014). Aproximación a las concepciones acerca de los trabajos prácticos en futuros docentes de ciencias naturales de la universidad Surcolombiana. Revista Tecné, 
Episteme y Didaxis: TED, Número Extraordinario. Memorias, Sexto Congreso Internacional sobre Formación de Profesores de Ciencias, Bogotá - Colombia. 1454-1463.

AMÓRTEGUI, E. y CORREA, M. (2012). Las Prácticas de Campo Planificadas en el Proyecto Curricular de Licenciatura en Biología de la Universidad Pedagógica Nacional. Caracterización desde la perspectiva del Conocimiento Profesional del Profesor de Biología. Bogotá: Fundación Francisca Radke y Universidad Pedagógica Nacional.

ANDERSON, D., THOMAS, G. P. y NASHON, S. (2009). Social barriers to meaningful engagement in biology field trip group work. Science Education, 93(3), 511-534. DOI:10.1002/ sce.20304.

BANET, E. (2000). La enseñanza y el aprendizaje del conocimiento biológico. En: F. Perales y P. Cañal (Eds.), Didáctica de las ciencias experimentales. Teoría y práctica de la enseñanza de las ciencias. Alcoy-España: Marfil.

BEREZUKI, P., OBARA, A. y SILVA, E. (2009). Concepções e práticas de professores de ciências em relação ao trabalho prático, experimental, laboratorial e de campo. Enseñanza de las Ciencias, Número Extra VIII Congreso Internacional sobre Investigación en Didáctica de las Ciencias, Barcelona, 2817-2822.

BERMÚDEZ, G., DE LONGHI,A., DÍAZ, S. y GAVIDIA, V. (2014). La trans- posición del concepto de diversidad biológica. Un estudio sobre los libros de texto de la educación secundaria española. Enseñanza de las Ciencias, 32 (3), 285-302. DOI: 10.5565/rev/ensciencias.1129.

BROMME, R. (1988). Conocimientos profesionales de los profesores. Enseñanza de las Ciencias, 6 (1), 19-29.

CAAMAÑO, A. (2003). Los trabajos prácticos en ciencias. En M. P. Jiménez (Coord.) Enseñar ciencias. Barcelona: Ed. Graó.

CALVO, G. (1995). La familia en Colombia -Un estado del arte de la investigación 1980-1994. Bogotá: Staf ICBF CARLSEN, W. (1999). Domains of Teacher Knowledge. In: J. GESSNEWSOME y N. LEDERMAN (Eds.). Examining Pedagogical Content Knowledge. The Construct and its Implications for Science Education. Dordrecht, Boston, London: Kluwer Academic Publishers.

CASTRO, A. y VALBUENA, E. (2007). ¿Qué biología enseñar y cómo hacerlo? Hacia una resignificación de la biología escolar. Tecné, Episteme y Didaxis, 22, 127-144.

CORREA, M. y VALBUENA E. (2012). Aproximación al estado del arte sobre los trabajos prácticos en la enseñanza de la biología: Hallazgos relacionados con los problemas de investigación durante el período (2004-2006). Revista Bio -grafía: Escritos sobre la Biología y su Enseñanza. 5 (8), 
117-135. DOI: 10.17227/20271034. vol.0num.0bio-grafia311.319.

COSTILLO, E., BORRACHERO, A., VILLALOBOS, A., MELLADO, V. y SÁNCHEZ, J. (2014). Utilización de la modelización para trabajar las salidas al medio natural en profesores en formación de educación secundaria. Revista Bio-grafía: Escritos sobre la Biología y su enseñanza. 7 (13), 165 - 175. DOI: 10.17227/20271034. vol.7num.13bio-grafia165.175.

CUTTER, E. (1993). Fieldwork: An essential component of biological training. Journal of Biological Education. 27 (1), 3-4. DOI:10.1080/002192 66.1993.9655292.

DEL CARMEN, L. (2000). Los trabajos prácticos. En J. Perales y P. Cañal (Coord.) Didáctica de las ciencias experimentales. Madrid: Alcoy.

DEL CARMEN, L. (2011). El lugar de los trabajos prácticos en la construcción del conocimiento científico en la enseñanza de la Biología y la Geología. En Cañal (Coord.) Didáctica de la Biología y la Geología. Barcelona: Graó.

DEL TORO, R.M. (2014). Concepciones y prácticas del profesorado acerca de las actividades de campo en educación secundaria de Biología en diferentes contextos educativos: los casos de Dinamarca, Campiñas (Sao Paulo, Brasil) y la comunidad de Madrid. (Tesis de Doctorado), Universidad Complutense de Madrid. Madrid, España.
DOURADO, L. (2006). Concepçoes e práticas dos profesores de ciencias naturais relativas à implementaçao integrada do trabalho laboratorial e do trabalho de campo. Revista electrónica de enseñanza de las ciencias. 5 (1), 192-212.

GAGLIARDI, R. (1986). Los conceptos estructurantes en el aprendizaje por investigación. Enseñanza de las Ciencias, 4 (1), 30-35.

GESS-NEWSOME, J. (2015). A model of teacher professional knowledge and skill including PCK: Results of the thinking from the PCK Summit. In: BERRY, A., FRIEDRICHESEN, P., LOUGHRAN, J. Re-examining Pedagogical Content Knowledge in Science Education. New York: Routledge.

GIL, D., CARRASCOSA, J., FURIÓ, C. y MARTÍNEZ-TORREGROSA, J. (1991). La enseñanza de las ciencias en la educación secundaria. Barcelona: ICE/Horsori.

GÓMEZ, M. (2014). Las prácticas de campo una estrategia didáctica para conservar el ecosistema de páramo desde el estudio ecofisiológico del frailejón (Asteraceae: Espeletia). Revista Bio-grafía: Escritos sobre la Biología y su Enseñanza. Edición Extraordinaria. DOI: 10.17227/20271034. vol.0num.0bio-grafia446.454.

GOULDER R., SCOTT, G.W. y SCOTT, L.J. (2013). Students' Perception of Biology Fieldwork: The example of students undertaking a preliminary year at a UK university, International Journal of Science 
Education, 35 (8), 1385-1406. DOI: 10.1080/09500693.2012.708796.

GRAHAM W., SCOTT, M.B., SCOTT L. y DEREK, C. (2015) Barriers To Biological Fieldwork: What Really Prevents Teaching Out of Doors? Journal of Biological Education, 49:2, 165-178. DOI: 10.1080/00219266.2014.914556.

GRANDI, L. y MOTOKANE, M. (2009). Reflexões sobre as características de um trabalho de campo que estimule a argumentação e a enculturação científica dos alunos. Enseñanza de las Ciencias, Número Extra VIII Congreso Internacional sobre Investigación en Didáctica de las Ciencias, Barcelona, 849-852.

GUARNIZO, M., PUENTES, O. y AMÓRTEGUI, E. (2015). Diseño y aplicación de una unidad didáctica para la enseñanza-aprendizaje del concepto diversidad vegetal en estudiantes de noveno grado de la institución educativa Eugenio Ferro Falla, Campoalegre, Huila. Tecné, Episteme y Didaxis. 37, 31-49. DOI: 10.17227/01213814.37ted25.45.

JACOB, F. (1999). La lógica de lo viviente. Una historia de la herencia. Traducción de Joan Senent y Rosa Soler. Barcelona: Tusquets.

JIMÉNEZ, M.P. (2003). La enseñanza y el aprendizaje de la biología. En: Jiménez, M.P. (Coordinadora) Enseñar ciencias. Barcelona: Graó.

JIMENEZ, A. (2006). El estado del arte en la investigación en las ciencias sociales. En: TORRES, A. y JIME-
NEZ, A. (2006) (compiladores). La práctica investigativa en ciencias sociales. Bogotá: Universidad Pedagógica Nacional.

JUDSON, E. (2011). The Impact of Field Trips and Family Involvement on Mental Models of the Desert Environment, International Journal of Science Education, 33:11, 1455-1472. DOI: 10.1080/09500693.2010.495758.

LAVIE ALON, N. y TAL, T. (2015). Student Self-Reported Learning Outcomes of Field Trips: The pedagogical impact, International Journal of Science Education, 37:8, 1279-1298. DOI:10.1080/09500693.2015.10347 97.

LEGARRALDE, T., VILCHES, A. y DARRIGRAN. G. (2009). El trabajo de campo en la formación de los profesores de Biología: Una estrategia didáctica para mejorar la práctica docente. II Jornadas de Enseñanza e Investigación Educativa en el campo de las Ciencias Exactas y Naturales, 28 al 30 de octubre de 2009, La Plata. Un espacio para la reflexión y el intercambio de experiencias. Disponible en: http://www.fuentesmemoria.fahce.unlp.edu.ar/trab eventos/ ev.560/ev.560.pdf

LOCK, R. (2010). Biology fieldwork in schools and colleges in the UK: An analysis of empirical research from 1963 to 2009, Journal of Biological Education, 44:2, 58-64. DOI:10.1080 /00219266.2010.9656195. 
MAHNER, M. y BUNGE, M. (2000). Fundamentos de Biofilosofía. México DF: Siglo XXI.

MANZANAL, R.F., RODRÍGUEZ, L.M. y CASAL, M. (1999). Relationship between Ecology Fieldwork and Student Attitudes toward Environmental Protection. Journal of Research in Science Teaching, 36, (4), 431-453. DOI:10.1002/ (sici)1098-2 736 (199904) $36: 4<43$ $1:$ : a i d - tea3>3.3.co;2-0.

MICK, N. (1996). Practical work in school biology evaluation, distortion, and deception. Journal of Biological Education. 30 (2), 83-87. DOI: 10.1080/00219266.1996.9655482.

MORAG, O. y TAL, T. (2012). Assessing Learning in the Outdoors with the Field Trip in Natural Environments (FiNE) Framework, International Journal of Science Education. 34 (5), 745-777. DOI:10.1080/09500693.201 1.599046 .

NUNES, I. y DOURADO, L. (2009). Concepções e práticas de professores de biologia e geologia relativas à implementação de acções de educação ambiental com recurso ao trabalho laboratorial e de campo. Revista electrónica de enseñanza de las ciencias. 8 (2), 671-691.

PARK, S. y CHEN, Y. (2012). Mapping Out the Integration of the Components of Pedagogical Content Knowledge (PCK): Examples From High School Biology Classrooms. Journal of Research in Science Tea- ching. 49 (7), 922-941. DOI:10.1002/ tea.21022.

RÍOS, M. y RUEDAS, M. (2009). El trabajo de campo: una estrategia para captar la complejidad de la realidad dirigida a futuros docentes en ciencias naturales. Enseñanza de las Ciencias, Número Extra VIII Congreso Internacional sobre Investigación en Didáctica de las Ciencias, Barcelona, 420-423.

RODRIGO, M., MORCILLO, J., BORGES, R., CALVO, A., CORDEIRO, N., GARCÍA, F. y RAVIOLO, A. (1999). Concepciones sobre el trabajo práctico de campo (TPC): Una aproximación al pensamiento de los futuros profesores. Revista Complutense de Educación. 10 (2), 261-285.

RODRÍGUEZ, C. y AMÓRTEGUI, E. (2012). Prácticas de campo como estrategia de enseñanza de la biodiversidad en alumnos de básica secundaria del colegio Champagnat: Planteamiento del problema. Revista EDUCyT Asociación Colombiana para la investigación en Educación en Ciencias y Tecnología EDUCyT, Vol. Extraordinario, diciembre.

SÁNCHEZ, P. y ESCOBAR, G. (2014). Las prácticas de campo para la enseñanza de la Paleobiología y su aporte al reconocimiento del patrimonio cultural y natural en educación secundaria del municipio floresta, Boyacá. Revista Bio-grafía Escritos: sobre la Biología y su Enseñanza. Edición Extraordinaria, 658-665. DOI: 
10.17227/20271034.vol.0num.0biografia658.665.

SHULMAN, L. (1986). Those who understand: Knowledge growth in teaching. Educational Researcher, 15 (2), 4-14.

SHULMAN, L. (1987). Knowledge and teaching. Foundations of the new reform. Harvard Educational Review. 57 (1), 1-21. DOI: 10.17763/ haer.57.1.j463w79r56455411.

STOLPE, K. y BJÖRKLUND, L. (2012). Seeing the Wood for the Trees: Applying the dual-memory system model to investigate expert teachers' observational skills in natural ecological learning environments, International Journal of Science Education. 34 (1), 101-125. DOI: 10.1080/09500693.2011.561505.

STORKSDIECK, M. (2006). Field trips in environmental education. Berlin: Berliner Wissenschafts-Verlag.

TAL, T. y MORAG, O. (2009). Reflective Practice as a Means for Preparing to Teach Outdoors in an Ecological Garden. Journal of Science Teacher Education. 20 (3), 245-262. DOI: 10.1007/ s10972-009-9131-1.
TAL, T., LAVIE ALON, N. y MORAG, O. (2014). Exemplary Practices in Field Trips to Natural Environments. Journal of Research in Science Teaching. 51 (4), 430-461. DOI: 10.1002/ tea.21137.

TARDIF, M. y LESSARD, C. (2014). $O$ oficio de profesor. História, perspectivas e desafios internacionais. Rio do Janeiro: Editora Vozes.

TILLING, S. (2004). Fieldwork in UK secondary schools: influences and provision. Journal of Biologi-cal Education. $\quad 38 \quad$ (2), 54-58. DOI:10.1080/00219266.2004.9655902.

VALBUENA, E. (2007). El conocimiento didáctico del contenido biológico. Estudio de las concepciones disciplinares y didácticas de futuros docentes de la universidad pedagógica nacional (Colombia). (Tesis de Doctorado), Universidad Complutense de Madrid, España.

VALBUENA, E., CORREA, M. y AMÓRTEGUI, E. (2012). La enseñanza de la Biología ¿un campo de conocimiento? Estado del arte. Tecné, Episteme y Didaxis. 31, 67-90. 
